\title{
Dynamical Evolutionary Psychology: Mapping the Domains of the New Interactionist Paradigm
}

\author{
Douglas T. Kenrick, Jon K. Maner, Jon Butner, \\ Norman P. Li, and D. Vaughn Becker \\ Department of Psychology \\ Arizona State University
}

\author{
Mark Schaller \\ Department of Psychology \\ University of British Columbia
}

\begin{abstract}
Dynamical systems and evolutionary theories have both been proposed as integrative approaches to psychology. These approaches are typically applied to different sets of questions. Dynamical systems models address the properties of psychological systems as they emerge and change over time; evolutionary models address the specific functions and contents of psychological structures. New insights can be achieved by integrating these two paradigms, and we propose a framework to begin doing so. The framework specifies a set of six evolutionarily fundamental social goals that place predictable constraints on emergent processes within and between individuals, influencing their dynamics over the short-term, and across developmental and evolutionary time scales. These social goals also predictably influence the dynamic emergence and change of cultural norms. This framework has heuristic as well as integrative potential, generating novel hypotheses within a number of unexplored areas at psychology's interface with the other biological and social sciences.
\end{abstract}

Every second-order interaction is moderated by third order interactions, which in turn are moderated by higher order interactions. Once we attend to interactions, we enter a hall of mirrors that extends to infinity. (Cronbach, 1975, p. 119)

Rather than focusing exclusively on factors inside the person or in the environment, psychology is an increasingly interactionist discipline. Psychologists now envision internal predispositions interacting with environmental inputs to affect ongoing thoughts and feelings in a continual and dynamic manner. These interactions unfold over time, as factors within the individual affect the choice and the interpretation of situations, and as individuals change their social situations. Conversely, factors in the situation not only trigger individual affective and cognitive inclinations in the short run, but over time, also change aspects of the person.

But Cronbach's (1975) quote (earlier) expresses a key concern about interactionism: How can a researcher enter this ever-changing array of interactions without getting lost in a "hall of mirrors?" If every interaction is moderated by a higher order interaction, perhaps it is pointless to isolate any particular factors in the person or

Requests for reprints should be sent to Douglas T. Kenrick, Dept. of Psychology, Arizona State University, Tempe, AZ 85287-1104. E-mail: douglas.kenrick@asu.edu situation for study. Indeed, the argument that human behavior is "too complex" for scientific study has been advanced in various forms over the years.

\section{Confronting Complexity: Dynamical Systems and Evolutionary Psychology}

Dynamical systems theory provides one pathway out of the hall of mirrors. This approach is concerned with complex multi-component systems as they change over time. To understand the balance of predators and prey, plants and animals, or parasites and hosts in a forest ecosystem, for example, one must observe the system over time. One of the exciting discoveries emerging from studies of complex systems is a ubiquitous tendency towards self-organization. Order often arises spontaneously-and sometimes suddenly - out of initially random interactions among components. Self-organization can occur within individuals' minds, as when coherent knowledge structures (e.g., self-concepts) emerge from the dynamic activation and inhibition of lower-level cognitions (Nowak, Vallacher, Tesser, \& Borkowski, 2000; Read \& Miller, 1998). Self-organization also occurs among groups of individuals, as in the emergence of status hierarchies, and across populations of individuals, as 
when clusters of shared beliefs and other cultural norms emerge from communication and influence among those individuals (Latané, 1996).

The logic of the dynamic perspective is intrinsically connected with the logic of evolutionary psychology (Kenrick, Li, \& Butner, in press). Both approaches are, for example, concerned with changes emerging over time in interconnected multi-component systems. Indeed, evolution by natural selection may be the paramount example of the power of self-organization. However, the two approaches have typically been applied to rather different questions. Dynamical systems models address questions about patterns of order and change within psychological and social systems (e.g., How do coherent self-concepts or social norms emerge out of initially disorganized components?) Dynamical models share with traditional cognitive psychology an emphasis on process, with less focus on content. Thus, they are rarely able to predict why self-concepts or social norms emerge with the one specific set of features rather than others. An evolutionary approach, on the other hand, is directly concerned with questions about specific content. By specifying particular adaptive problems bearing on the survival and reproduction of ancestral organisms, evolutionary models yield hypotheses specifying that certain cognitive and behavioral patterns are more likely than others to be widespread within human populations.

The mutual implications of dynamical and evolutionary approaches to cognition and behavior have yet to be conceptually or empirically explored within the behavioral sciences. In this article, we map out a general integrative framework that may be useful in identifying questions and generating novel hypotheses about the dynamic structures that emerge within human minds, in social interaction, and across human populations.

We begin by considering one key insight from evolutionary psychology: The adaptive problems faced by ancestral humans have given rise to a corresponding set of fundamental psychological goals that guide contemporary human cognition and behavior within specific domains of social life. These adaptive goals have consequences for social dynamics at several levels: affecting short-term patterns, changes in cognitive structures across individuals' lives, and evolutionary changes in the cognitive and behavioral features of populations. Finally, we discuss how these processes that operate on individuals can also have predictable consequences on the emergent properties of the shared beliefs and social norms that define human cultures.

\section{The Domains and Goals of Social Life}

Other people present us with multitudinous informational inputs. Some are tall, some short; some are female, some male; some are relatives, others friends, and still others strangers. The need to make quick, efficient, and functional decisions makes it impossible to attend equally to every piece of information. From an evolutionary perspective, cognitive processes involve adaptive selectivity: We attend to the information most relevant to important domain-specific goals. And our responses to that information are informed by heuristic decision-rules that, in ancestral environments, would have been functionally adaptive (i.e., facilitated reproductive success).

Evolutionary reasoning-bolstered by neurophysiological evidence (Panksepp, 1982) — suggests a finite set of fundamental human goals, each linked to an adaptive problem posed by the environments in which ancestral humans lived. What are the pre-eminent domains of adaptive problems confronting humans living in social groups? Based on several reviews of literature related to this question (Bugental, 2000; Buss, 1999; Fiske, 1992; Kenrick et al., in press), we identify six key domains of social life associated with corresponding fundamental goals. The satisfaction of each goal serves the ultimate function of passing on one's genes to future generations, and does so by facilitating one or more of the following outcomes: increasing one's own reproductive life-span; maximizing the number of productive mating opportunities; or ensuring that one's offspring reach reproductive age themselves. The goals are:

(1) Self-protection. A necessary precondition to successful reproduction is to stay alive and healthy. This requires psychological mechanisms that motivate vigilant avoidance of individuals who pose threats to one's health. The self-protective goal is associated with emotions that motivate avoidant behaviors (e.g., fear, disgust), and promote selective attention to threat-relevant features of others (e.g., large outgroup males).

(2) Coalition formation. Health and reproductive fitness is promoted not only by the avoidance of threats, but also by building coalitional bonds to promote cooperation and mutual assistance in times of need. This coalitional goal may be linked to emotions (such as empathy) that motivate pro-social behavior and may promote selective attention to information (such as similarity or past evidence of reciprocity) indicating which other people are good bets for the expenditure of assistance.

(3) Status-seeking. For both sexes, there are adaptive advantages to gaining and maintaining social status, including greater access to material resources and extended social alliances. Because females use male status as a cue for mate selection, males are more likely to be concerned with possible loss of status. This goal is likely to promote selective attention to size, sex, and other indicators of relative social status (some of which may be learned and culture-specific). 
(4) Mate choice. Access to viable mates is essential to reproductive success; so individuals are likely to selectively attend to features connoting reproductive viability. Because of inherent differences in amount and type of parental investment, males and females are likely to attend to somewhat distinct sets of features in the other sex. For instance, women may attend more to indicators of status and overall genetic fitness, and men to age and other indicators of fertility.

(5) Relationship maintenance. Because human infants are helpless and slow to develop, their survival is enhanced by the presence and support of both parents. Thus, long-term cooperative mating relationships would have been adaptive. This goal may direct attention to information relevant to decisions about relationship maintenance, including attractiveness of same-sex interlopers on the social horizon and the ratio of females to males in the local mating pool.

(6) Offspring Care. Because parental care is critical to offspring survival, it is an essential goal itself. The need for parental care is not constant across time or context; so individuals are likely to selectively attend to information that inform decisions about the devotion of resources to specific offspring. These include the presence of other offspring, age of offspring, care provided by other kin, and immediate dangers to offspring.

Each of these goals is also associated with a specific social geometry that constrains the nature of interpersonal interactions. Self-protective goals lead to the emergence of mutually exclusive social categories that cleave the world according to distinctions between individuals who are in our group and those who are not. Cooperation goals lead to the emergence of overlapping circles of friends and relatives. Status-seeking goals demand the emergence of hierarchical arrangements. Mating relationship goals demand interactions at the dyadic level; and so on (see Figure 1; for elaboration, see Kenrick et al., in press).

Thus, the geometries of social life, which govern interaction and social influence- - and so are fundamental to the dynamic emergence of cultural norms and other aspects of socially-shared cognition (Latané \& L'Herrou, 1996) — are themselves constrained by the psychological products of human evolutionary history. This hints at just one of the useful implications that can arise when considering the implications of evolutionary psychology on the predictions generated by dynamical systems models. Some of these implications are explored more fully by Kenrick et al. (in press). Here, we turn our attention to a broader set of implications that appear when considering the influence of evolutionarily based goals on the dynamic emergence of social phenomena.

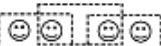

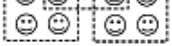

Coalition Formation

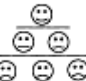

Status

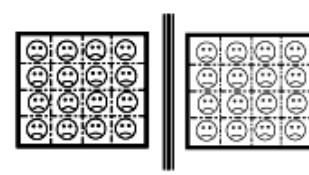

Self-Protection

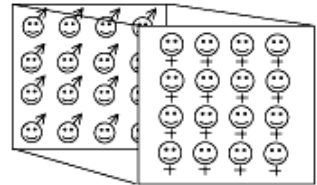

Mate Choice

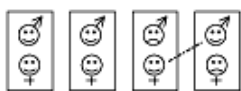

Relationship Maintenance

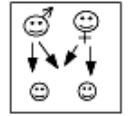

Parental Care

Figure 1. Different decision rules for each social problem domain lead to the emergence of different social geometries (based on Kenrick, Li, \& Butner, in press).

\section{An Organizing Framework For Dynamical Evolutionary Psychological Questions}

Table 1 presents a conceptual framework for organizing the questions that can be addressed by an evolutionarily informed dynamical social psychology.

One dimension of the table refers to the timeframe of the dynamic process. Some dynamic processes occur over short time spans-as patterns emerge within moment-to-moment and hour-to-hour thoughts, feelings, and social behaviors. Dynamic processes also continue to unfold over the course of individuals' lives, and so guide development and change in personality, social cognition, and social interactions throughout the lifespan. Finally, dynamic processes operate on populations over a much longer, evolutionary timeframe, and so have influenced the nature and organization of cognitive and behavioral mechanisms common across human populations.

Dynamic evolutionary questions can be divided further into those dealing specifically with interactions among components inside the organism (e.g., the dynamic relations between attention, thought, and competing functional motivations), and those that involve interactions between organisms (e.g., the emergence of 
Table 1. A Framework for Organizing Questions Arising From a Dynamical Evolutionary Psychology.

\begin{tabular}{|c|c|c|c|}
\hline \multirow[b]{2}{*}{ Units } & \multicolumn{3}{|c|}{ Time Frame } \\
\hline & Short-term & Developmental & Evolutionary \\
\hline Within Person & $\begin{array}{l}\text { How do coherent psychological } \\
\text { structures emerge from the dynamic } \\
\text { interaction of affective and cognitive } \\
\text { processes? } \\
\text { Example: Activating self-protection vs. } \\
\text { mating motive re-organizes attentional } \\
\text { and perceptual processes. }\end{array}$ & $\begin{array}{l}\text { How does the internal organization of } \\
\text { an individual's cognitions, traits, and } \\
\text { other characteristics change over the } \\
\text { lifespan? } \\
\text { Example: Relationship between } \\
\text { aggressive behavior and testosterone } \\
\text { levels changes at puberty. }\end{array}$ & $\begin{array}{l}\text { How do constellations or syndromes of } \\
\text { traits co-evolve over generations? } \\
\text { Example: Inherent pattern linking } \\
\text { female reproductive physiology, } \\
\text { parenting behavior, and attitudes about } \\
\text { casual sex. }\end{array}$ \\
\hline Between People & $\begin{array}{l}\text { How do the interactions between } \\
\text { individuals in interconnected networks } \\
\text { self-organize into group level patterns? } \\
\text { Example: Mutual cooperation or } \\
\text { conflict in a social dilemma situation. }\end{array}$ & $\begin{array}{l}\text { How do social groups organize and } \\
\text { re-organize themselves over the } \\
\text { life-spans of the individuals involved? } \\
\text { Example: Dynamics between sexes } \\
\text { change at puberty. }\end{array}$ & $\begin{array}{l}\text { How does the local population of types } \\
\text { mutually constrain one another? } \\
\text { Example: Physical and behavioral } \\
\text { characteristics of males co-evolve with } \\
\text { those of females. }\end{array}$ \\
\hline
\end{tabular}

group norms). This distinction comprises the second dimension of this conceptual framework, yielding a scheme in which research questions can be classified into one of six conceptual categories.

Of course, moment-to-moment interactions are necessary parts of the never-ending dynamic story of human developmental change, and of evolutionary change across time. And, in social species, there is an intrinsic connection between intra-individual dynamics and the dynamic interactions between individuals. Hence, the processes identified within each category are necessarily related to processes identified within other categories as well. The general framework is nevertheless useful for framing a set of questions, and suggesting a set of novel hypotheses, about the psychological structures that emerge dynamically within different domains of social life.

\section{Short-term Interactions of Components Within The Person}

In any given moment, the brain processes millions of neural inputs regarding temperature, light, smell, bodily position, respiration, and so on. Conscious experience represents a patterned organization of sensation, attention, perception, categorization, memory, affect, and plans for behavioral action. There have been a number of dynamical analyses of psychological phenomena at the individual level. For example, Nowak et al. (2000) examined how self-organization of beliefs, attitudes, and memories gives rise to coordinated change, differentiation, and global coherence in one's self-concept. From an evolutionary dynamic perspective, one might ask: In what ways are self-concepts and other emergent knowledge structures constrained? Are some types of information more likely than others to define these structures?

An evolutionary perspective inclines one to ask how the various components of the individual are differentially organized to meet different adaptive goals. Dy- namically constructed knowledge structures pertaining to the social world may be drawn to a limited number of attractor states that correspond to fundamental adaptive goals. For instance, stereotypic impressions of groups often coalesce around traits connoting either friendliness or competence (Fiske, Cuddy, Glick, \& $\mathrm{Xu}$, in press)—dimensions relevant to self-protection, coalition-building, and status-seeking goals. Personality traits are also perceptually organized in ways that correspond to specific goals linked to the adaptive problems identified earlier (e.g., Read, Jones, \& Miller, 1990; Roberts \& Robins, 2000). The same constraints on content may apply to the self-concept. The traits likely to define self-concepts most stronglythose most accessible and subjectively important-are likely to be those corresponding to evolutionarily fundamental goals.

The defining contents of knowledge structures are, of course, in constant flux. Evidence bearing on the dynamic activation-and-inhibition processes of human cognition suggests a sort of winner-take-all state of psychological affairs, in which only one executive system predominates at a given time (Martindale, 1991). This implies that the activation of one adaptive motivational system inhibits others, with important consequences for the specific knowledge structure activated at any given time. For example, a perceived physical threat will activate a self-protective goal, leading threat-relevant features of self and other (e.g., familiarity, physical strength) to become especially salient, while features pertaining to potential mating opportunities fade into the background. On the other hand, if a mating goal is activated, mating-relevant features of self and other (e.g., physical attractiveness) become salient, while self-protection related information diminishes in import.

An evolutionary perspective implies that some domain-specific goals will take priority over others. For example, one failure of self-protection has greater functional consequences than one failure in mate-seek- 
ing (a woman who foregoes a mating opportunity to save herself from bodily harm will live to see other mating opportunities, but the reverse may not be true). These examples illustrate how intra-person dynamics are intrinsically linked to inter-personal dynamics.

\section{Short-term Interactions Between Individuals}

Individual decisions have implications for social behaviors, which in turn lead to dynamic processes at the inter-personal level (Latané, 1996; Nowak, Szamrej, \& Latané, 1990). These interpersonal interactions are likewise constrained by evolutionarily based goals.

These goals are not insensitive to the environment. From an evolutionary perspective, they can be conceived of as decision rules, or if-then statements, responsive to inputs from other individuals with whom one interacts (Cosmides \& Tooby, 1992; Kenrick et al., in press). We conducted a series of spatial simulations to examine implications of individual adaptive decision rules at the dynamic level (Kenrick et al., in press). One series explored how individual differences in decision rules for cooperation versus competition affected community level outcomes. A few individuals with unusual decision rules (such as a very low threshold for aggression) could change outcomes for whole communities, depending on the initial random distribution of neighbors (see Figure 2).

A second series of simulations explored the mutual impact of mating decision rules, using a two-layer geometry similar to that for mate choice depicted in Figure 1. A given female's decision rule for sexually restricted or unrestricted behavior was keyed to the behaviors of males in her immediate vicinity. Conversely, males' decision rules were keyed to the behaviors of contiguous females. Consistent with empirical data and theory, females' decision rules were assumed to lean somewhat toward a restricted mating strategy, preferring this option unless an overwhelming majority of local males were unrestricted. Males, on the other hand, were assumed to lean slightly toward an unrestricted strategy, but to behave in a restricted manner if a majority of local females did so. The mutual feedback between men and women-coupled with the different decision rules employed by each sex-had nonlinear consequences on the actual mating decisions made by the individuals within this simulated community. Using normal decision rules, both men and women converged into communities that were predominantly restricted; whereas relatively small changes in the rules adopted by either sex had rather larger consequences at the community level. For example, if females use typically "male" rather than "female" decision rules, the same initial communities tend to converge into clusters of individuals acting mostly in an unrestricted manner.
These simulations help clarify the difference between underlying traits and overt behavioral manifestations. In the simulations, males' behavior varied depending on females' decision-rules, even though the males' underlying dispositions did not change. Such simulations demonstrate how different local ecologies may induce individuals with a given trait to act very differently, even though their underlying traits do not vary. For a real world parallel, homosexual and heterosexual males are, in most ways, very similar in mating preferences, but homosexual males are strikingly more unrestricted than heterosexuals (Bell \& Weinberg, 1978; Kenrick, Keefe, Bryan, Barr, \& Brown, 1995). This dif-
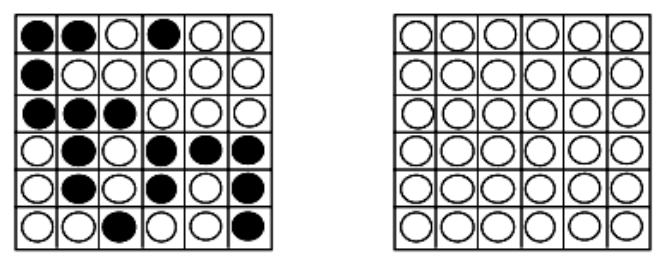

$\mathbf{A}$
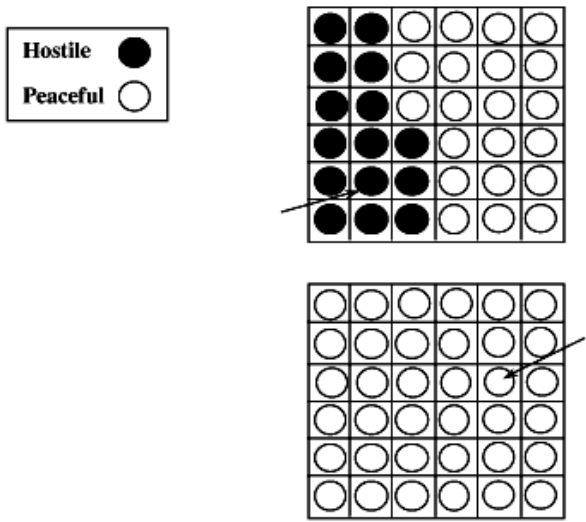

C

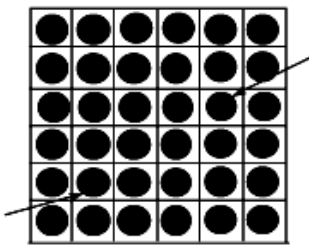

D

Figure 2. The neighborhood at the left depicts individuals acting either aggressively (dark) or peacefully (white). If all individuals attempt to match the majority of their neighbors, this neighborhood will self-organize into a totally peaceful neighborhood (as shown at the right top). If some individuals have very low thresholds for aggression (as marked), this can change outcomes for the whole neighborhood, depending on their location and random variations in their neighbors' behaviors (based on Kenrick, $\mathrm{Li}, \boldsymbol{\&}$ Butner, 2001). In these examples, the marked individuals will act aggressively if any of their neighbors were hostile on the previous round of interaction. In $\mathbf{B}$, one aggressive individual radically changes the outcome for the whole community. In C, a single low threshold individual has no impact on the eventual outcome, but in combination with the other low-threshold individuals, this individual completely reverses the outcome for the neighborhood as a whole (the contingency involving this individual's impact demonstrates the critical importance of initial conditions in complex systems). 
ference may reflect the dynamic implications of slightly different local ecologies, rather than an underlying difference in decision-rules for restricted behavior. If heterosexual males' intended partners used the same decision-rules as they do, predominantly unrestricted communities would likely be much more frequent.

Similar discontinuities between individual predispositions and overt behaviors are likely in other fundamental domains of social life. The discovery of these non-obvious outcomes demands attention to the dynamic interactions between individuals, and to the specific social geometries that govern their interactions within the different domains.

Specific hypotheses might be deduced and tested in a variety of ways. Empirical data from individuals can provide necessary information about the decision criteria for different sorts of interactions within different types of social geometries. This information about individual decision rules can be used to set the parameters of simulations designed to model dynamic implications over time. These models may reveal further non-obvious consequences resulting from the dynamic interplay of decision rules. Those results can then be tested empirically against sociometric data and results of new experiments.

\section{Within-Person Dynamics Over The Life Course}

Patterns of individual cognition, affect, and behavior can change over the life course. Some of these changes may be relatively sudden - the sort of dramatic discontinuity that suggests nonlinear dynamic interactions within the individual. Evolutionary biologists sometimes view developmental processes through the lens of a life history - a genetically organized plan for allocating energy to survival, growth, and reproduction (Kenrick \& Keefe, 1992). Human life histories are punctuated by several substantial discontinuities. Life begins with a lengthy period of somatic effort, which ends in dramatic physiological, cognitive, and affective changes at puberty. Then, a large increase in production of reproductive hormones is accompanied by increases in attention to the opposite sex, concern with social comparison within sex, increased probabilities of risk-taking behaviors, and so on. For females, there is another disjunctive change at menopause, when changes in hormone production accompany dramatic physical, cognitive, and affective changes.

Individuals sometimes undergo other sudden psychological changes - in attitudes and values-in concert with changes in the social environment (e.g., disrupted mating relationship; entry into a new circle of friends). These sudden changes may be most likely to occur on attitudes and values most important to individuals' self-concepts (Latané \& Nowak, 1994). A dynamical approach to development suggests that indi- viduals are drawn toward different psychological attractor states at different points in the lifespan; and the logic of evolutionary theory further suggests that these states are likely to correspond to the fundamental functional goals of social life.

Studies of other species reveal the possibility of alternative strategies for solving the same adaptive problem at significant stages in an organism's life history. For example, some male salmon develop to full size and battle with one another for reproductive access to females; others develop very large testes within much smaller bodies, hide in the gravel near nesting pairs, and dart in to release their own sperm when the female releases her eggs (Gould \& Gould, 1989). The dynamics of human development may lead to parallel alternative tracks through the evolutionarily meaningful domains of human life. For instance, research on social dilemmas suggests that some individuals adopt a chronically cooperative strategy, whereas others adopt a habitually competitive style (VanLange, DeBruin, Otten, \& Joireman, 1997). Similarly, there are chronic individual differences in the probability of adopting a restricted or unrestricted mating strategy (Gangestad \& Simpson, 2000). The adoption of one strategy versus another may result from the nonlinear dynamical interplay of existing traits (physical features, temperament, etc.). Slight developmental changes in these underlying traits and cognitions may tip the balance toward one of several distinct, and self-maintaining, behavioral trajectories. These self-maintaining trajectories can be conceived, in dynamic terms, as developmental attractor states.

Pursuing this line of reasoning can generate novel research questions. How might personality and self-concept become dynamically re-organized as a consequence of changes in some single within-person component (e.g., change in sex drive)? How might these changes be constrained by the evolutionary goals most relevant to particular stages in human life histories (e.g., mate choice vs. offspring care)? How do changes in the internal organization of traits bearing on one social domain (such as status-seeking or mate-choice) connect dynamically to changes in traits relevant to other domains (such as self-protection or coalition formation)?

\section{Between-Person Dynamics Over the Life Course}

Life-history strategies, with their associated internal organization of psychological components and external organization of morphology, are intrinsically connected to social dynamics. Consider the Nassau grouper. In this species, all young individuals are females who join harems controlled by a large territorial male. When the male dies, the largest female goes through a series of hormonal and morphological changes and becomes a male (Warner, 1984). Thus, the 
self-organization of traits in this species is, in a dramatic way, influenced by events in the local social environment, and in turn exerts a reciprocal influence on other individuals in that environment. Similarly, interactions within the local social environment may influence the internal psychological make-up of individuals within that environment, and lead to developmental effects that have additional consequences for other people in the local social network. One example may be provided by research revealing effects of family dynamics on timing of pubertal maturation in girls (Ellis, McFadyen-Ketchum, Dodge, Pettit, \& Bates, 1999).

Over the lifespan, there are likely to be unique dynamic consequences associated with the different sorts of interpersonal interactions that define the various domains of social life. Some of these consequences may reflect particular dynamic patterns of behavior relevant to the specific social geometries associated with each domain. Within coalition networks, cooperative cycles of behavior may become deeply entrained among some individuals, whereas others - those who are excluded from coalitions for whatever reason-may adopt different, less cooperative strategies to gain access to valued resources. These group dynamics, once in place, can resist the influence of single individuals and so can be remarkably difficult to change (Plous, 1985). There are different dynamic consequences associated with mating relationships. When individuals forge long-term mating relationships, they essentially remove themselves from the pool of available mates. This can affect factors linked to the social geography of mate-choice (e.g., sex ratio), which have substantial influences on others' mating decisions (Guttentag \& Secord, 1983). Even small changes in these parameters can have substantial effects. Hence, one individual's decision to enter or leave a mating relationship can have unanticipated consequences on the behavior of many others within the social environment (e.g., leading others to enter or exit mating relationships, in turn influencing the trajectory of still other peoples' life histories).

\section{Within-Person Dynamics over the Evolutionary Time Course}

Social and personality psychologists customarily consider the events that take place from moment to moment in individuals' lives, and are also accustomed to inquiry into the developmental changes that occur over the course of individuals' lives. Psychologists are less likely to inquire into the evolutionary changes that occur in biological populations over tens of thousands of years. Nevertheless, informed speculations about human evolutionary history can help generate novel insights about contemporary psychological processes. In this spirit, it is worth speculating about the effects that within-person dynamics might have had on the evolution of human populations, and the ways in which those dynamics can be constrained by the existence of fundamental goals.

For a given organism attempting to make its way in the world, some suites of characteristics will mesh better than others. Genes (and their phenotypic expressions) are selected if they are compatible with the collection of other genes making up prototypical individuals in the local population. Consider the domain of parental care. Male and female mammals have evolved different sets of inter-linked characteristics. A female mammal's reproductive physiology, her approach to choosing a mate, and her parenting behavior all tie together. Females can carry fetuses and nurse them after birth; males can not. Associated with these behaviors are hormonal differences. Females produce oxytocin and progesterone, which influence not only physical capacities-such as milk production-but also parenting behaviors (Zeifman \& Hazan, 1997). Males produce fewer of these hormones, but more testosterone, which is associated with another interlinked complex of bodily changes and behaviors, including enlarged upper body muscles, deeper voices, and greater inclinations toward aggressive competition (Mazur \& Booth, 1998).

This example illustrates processes relevant to one particular fundamental domain of social life. Other such co-evolutionary processes are likely to have occurred within the other domains. In the status domain, for example, individual differences in testosterone levels and physical size are dynamically linked to dominant and aggressive behavioral inclinations in an evolutionarily adaptive way (Mazur \& Booth, 1998). By inquiring into the processes unique to each domain, a richer understanding of human evolutionary history is likely to emerge, and along with it, a richer set of hypotheses about contemporary cognition and social behavior.

\section{Between-Person Dynamics over the Evolutionary Time Course}

Over generations, natural selection shapes organisms with sets of characteristics that promote survival within the dynamic context of other organisms they are likely to encounter. Thus, co-evolutionary processes often link the evolution of one class of organisms to the evolution of other classes of organisms. A simple example involving multiple species is the co-evolution of parasites and hosts. Similar co-evolutionary processes can occur within one species, as in the mutual constraints provided by female and male behavioral tendencies. Similarly, psychological mechanisms related to cheating and cheater-detection, or to aggression and fear may have evolved in dynamic tandem. The evolution of communication systems provides a clear example. For an emotional expression of anger to function as a warning, the communicator must have the capacity to transmit the appropriate cues, and the intended re- 
cipient must have the capacity to receive and interpret those cues.

It will be useful to consider how human interpersonal behavior-driven by the desire to satisfy specific goals in specific domains - constrained the co-evolution of cognitive mechanisms and behavioral tendencies. To do so in a rigorous way demands that we place our inquiries into human social cognition within the larger tradition of inquiry into the evolutionary histories of other social species. For example, behavioral ecologists have explored questions about whether large brain size among primates is associated with living in large social groups, and whether sex differences in physical size are related to a species' mating arrangements (e.g., Dixson, 1999; Dunbar, 1993). Research on dynamics in the different domains of human social life can be made richer by this comparative perspective, and psychologists are in a unique position to raise new cross-disciplinary questions yet to be considered by researchers focused on other species.

\section{Additional Implications: Evolutionary Influences on the Dynamic Emergence of Culture}

Thus far, we have discussed how adaptive goals conspire with dynamical processes to shape the patterns and structures of human social cognition and behavior. There are psychological implications at the sociological level-as evolved decision-rules are intrinsically linked to the patterns and structures of human culture. The very existence of culture-socially-shared belief systems and norms-depends crucially on the dynamic social influence processes precipitated by interpersonal communication across social landscapes (Harton \& Bourgeois, in press; Latané, 1996). Processes of interpersonal communication-decisions about what to say and how to say itare influenced by a variety of motives and goals. Thus, the specific features and contents of emergent cultural norms are inevitably influenced by the same goals (Schaller \& Conway, 1999, 2001). If the goals that most govern individuals' cognitions and decision-rules are those pertaining to essential adaptive problems, then those same goals are likely to affect the dynamical processes through which cultural norms emerge and change over time (c.f., Tooby \& Cosmides, 1992).

On this theme, Krebs and Janicki (in press) describe the ways in which moral norms within cultures may have emerged as solutions to specific adaptive problems. This evolutionary analysis of moral norms is consistent with Haidt's (2001) analysis of moral reasoning, which suggests that judgments of morality may be based on evolved intuitive preferences rather than on rational analysis. These evolutionary analyses of moral norms could be enriched by a consideration of the dynamical processes through which interacting individuals influence each other. Other theoretical analyses - abetted by computer simulations - have focused more explicitly on the ways in which emerging cultural norms depend on the interpersonal dynamics of social influence (Axelrod, 1986; Kenrick et al., in press). Results of these simulations reveal that particular cultural norms emerge, while others do not, depending on a dynamic interplay of different individuals' decision rules_-rules rooted in evolutionarily basic concerns with self-protection, coalition-formation, and mate-selection.

These promising conceptual analyses suggest that a dynamical evolutionary perspective may offer a rich source of answers to important questions about the origins, persistence, and change of human cultures. For instance: Why do some beliefs spread quickly and widely throughout a human population-and so become cultural norms-while others do not? An evolutionary perspective suggests that those beliefs most pertinent to fundamental adaptive problems are most likely to become normative, while those that are less pertinent may remain merely idiosyncratic. Empirical inquiry into the transmission of "urban legends" reveals results consistent with this evolutionary analysis. For example, legends arousing greater disgust (and so immediately relevant to protection against disease) are more likely to be communicated between individuals, and so ultimately are more likely to become part of a cultural mythology (Heath, Bell, \& Sternberg, 2001).

Another promising lead is provided by research into the emergence of socially shared stereotypes. Examining dynamic processes through which individual goals and interpersonal communication shape shared stereotypic beliefs, Schaller and Conway (1999) found that these effects occurred more strongly on beliefs pertaining to aggression than on beliefs pertaining to intelligence. This asymmetry is consistent with a consideration of evolved function, as aggression is more critically related to the fundamental adaptive problems of self-protection arising in intergroup contexts. Another set of studies (Schaller, Conway, \& Tanchuk, 2002) revealed that, in general, those traits most likely to be talked about in interpersonal contexts are also most likely to persist in the culturally-shared stereotypes of prominent ethnic groups. These results beg the question of why some traits are more communicable than others. One set of testable possibilities is provided by the evolutionary approach: Those traits that most clearly convey information related to essential adaptive problems and social goals (self-protection, coalition-formation, mate-selection, etc.) are most likely to comprise the contents of interpersonal communications, and so are most likely to define the emergent contents of socially-shared stereotypes that persist in a culture across time.

These are just a few examples of the ways in which evolutionary goals may influence the dynamics of in- 
terpersonal communication and, ultimately, the contents of cultural norms. By more fully exploring the ways in which evolutionary goals influence the interpersonal communication of the many beliefs and behaviors that define cultures, we may arrive at a more fully informed understanding of the evolutionary and psychological foundations of culture itself.

\section{Conclusion: Out of the Hall of Mirrors and Into the Map Room}

Social processes involve a continual dynamic interaction between individual cognition and the social and physical environment. These interactions are, at first blush, as overwhelming and confusing as a hall of mirrors is to a young child lost in an amusement park. To discern pattern and organization within this bewildering complexity, a broad integrative perspective is essential.

Dynamical systems theory provides one tool to help us search for those patterns. Dynamical models have revealed the ubiquity of self-organization in nature, and uncovered analogous patterns in systems as widely ranging as genes, neurons, human neighborhoods, and economic markets. What an evolutionary analysis adds to the dynamic perspective is a focus on content. By focusing on adaptive content, we should be able to make more specific predictions about which self-organizing structures and patterns will emerge within human minds and across social landscapes. This focus on content can add useful predictive boundaries on the application of familiar dynamic models to psychological phenomena. The most interesting and important patterns that emerge from dynamic interactions within and between individuals are likely to be those emerging within the evolutionarily fundamental domains of social life. An integrated dynamical evolutionary psychology can offer an especially powerful set of conceptual insights. These insights can help us deduce the psychological and cultural implications of an intrinsically dynamic natural world where genes interact continually and bidirectionally with other genes, neurons interact with other neurons, cognitive mechanisms interact with affective mechanisms, predators interact with prey, and so on.

We have focused primarily on processes of self-organization and emergent coherence to illustrate this assertion, but the same assertion is likely to apply also to other prototypic phenomena associated with dynamical systems, such as cusp catastrophes. It is perhaps no mere coincidence that Tesser and Achee (1994) used the conflict between mating and social pressure to illustrate the relevance of the cusp model to social behavior, and that Zeeman (1976) used the conflict between anger and fear in a self-protection situation.

The map we have outlined here for an evolutionary dynamical psychology is both speculative and incom- plete. In cartographic terms, it is an initial sketch of a possible coastline, with little detail of what lies within. There are hints here about where to find good inlets, but the largest expanse of potential discoveries lies ahead. What is exciting about this sketchy map is that it promises a theoretical continent that invites multi-disciplinary exploration. The realm of dynamical evolutionary psychology has the potential to encompass and unite all the local terrain already explored by psychologists, anthropologists, ethologists, and other behavioral researchers.

\section{References}

Axelrod, R. (1986). An evolutionary approach to norms. American Political Science Review, 80, 1095-1111.

Bell, A. P., \& Weinberg, M. S. (1978). Homosexualities: A study of diversity in men and women. New York: Simon \& Schuster.

Bugental, D. B. (2000). Acquisition of the algorithms of social life: A domain-based approach. Psychological Bulletin, 126, 187-219.

Buss, D. M. (1999). Evolutionary psychology: The new science of the mind. Boston: Allyn \& Bacon.

Cosmides, L., \& Tooby, J. (1992). Cognitive adaptations for social exchange. In J. Barkow, L. Cosmides, \& J. Tooby (Eds.), The adapted mind (pp. 163-228). New York: Oxford University Press.

Cronbach, L. J. (1975). Beyond the two disciplines of scientific psychology. American Psychologist, 30, 116-127.

Dixson, A. F. (1999). Primate sexuality: Comparative studies of the prosimians, monkeys, apes, and human beings. Oxford, England: Oxford University Press.

Dunbar, R. (1993). Coevolution of neocortical size, group size, and language in humans. Behavioral \& Brain Sciences, 16, 681-735.

Ellis, B. J., McFadyen-Ketchen, S., Dodge, K. A., Pettit, G., \& Bates, J. (1999). Quality of early family relationships and individual differences in the timing of pubertal maturation in girls: A longitudinal test of an evolutionary model. Journal of Personality and Social Psychology, 77, 387-401.

Fiske, A. P. (1992). The four elementary forms of sociality: Framework for a unified theory of social relations. Psychological Review, 99, 689-723.

Fiske, S. T., Cuddy, A. J. C., Glick, P., \& Xu, J. (in press). A model of (often mixed) stereotype content: Competence and warmth respectively follow from perceived status and competition. Journal of Personality and Social Psychology.

Gangestad, S. W., \& Simpson, J. A. (2000) The evolution of human mating: Trade-offs and strategic pluralism. Behavioral and Brain Sciences, 23, 573-644.

Gould, J. L., \& Gould, C. L. (1989). Sexual selection. New York: Scientific American Library.

Guttentag, M., \& Secord, P. F. (1983). Too many women? The sex ratio question. Beverly Hills, CA: Sage.

Haidt, J. (2001). The emotional dog and its rational tail: A social intuitionist approach to moral judgment. Psychological Review, $108,814-834$.

Harton, H. C., \& Bourgeois, M. J. (in press). Cultural elements emerge from dynamic social impact. In M. Schaller \& C. S. Crandall (Eds.), The psychological foundations of culture. Mahwah NJ: Lawrence Erlbaum Associates, Inc.

Heath, C., Bell, C., \& Sternberg, E. (2001). Emotional selection in memes: The case of urban legends. Journal of Personality and Social Psychology, 81, 1028-1041. 
Kenrick, D. T., \& Keefe, R. C. (1992). Age preferences in mates reflect sex differences in reproductive strategies. Behavioral and Brain Sciences, 15, 75-133.

Kenrick, D. T., Keefe, R. C., Bryan, A., Barr, A., \& Brown, S. (1995). Age preferences and mate choice among homosexuals and heterosexuals: A case for modular psychological mechanisms. Journal of Personality \& Social Psychology, 69, 1166-1172.

Kenrick, D. T., Li, N. P., \& Butner, J. (in press). Dynamical evolutionary psychology: Individual decision rules and emergent social norms. Psychological Review.

Krebs, D. L., \& Janicki, M. (in press). Biological foundations of moral norms. In M. Schaller \& C. S. Crandall (Eds.), The psychological foundations of culture. Mahwah NJ: Lawrence Erlbaum Associates, Inc.

Latané, B. (1996). Dynamic social impact: The creation of culture by communication. Journal of Communication, 46, 13-25.

Latané, B., \& L'Herrou, T. (1996). Spatial clustering in the conformity game: Dynamic social impact in electronic groups. Jour nal of Personality and Social Psychology, 70, 1218-1230.

Latané, B., \& Nowak, A. (1994). Attitudes as catastrophes: From dimensions to categories with increasing involvement. In R. R. Vallacher \& A. Nowak (Eds.), Dynamical systems in social psychology (pp. 219-249). San Diego, CA: Academic.

Martindale, C. (1991). Cognitive psychology: A neural-network approach. Pacific Grove: CA: Brooks/Cole.

Mazur, A., \& Booth, A. (1998). Testosterone and dominance in men. Behavioral and Brain Sciences, 21, 353-397.

Nowak, A., Szamrej, J., \& Latané, B. (1990). From private attitude to public opinion: A dynamic theory of social impact. Psychological Review, 97, 362-376.

Nowak, A., Vallacher, R. R., Tesser, A., \& Borkowski, W. (2000). Society of self: The emergence of collective properties in self-structure. Psychological Review, 107, 39-61.

Panksepp, J. (1982). Toward a general psychobiological theory of emotions. Behavioral and Brain Sciences, 5, 407-467.

Plous, S. (1985). Perceptual illusions and military realities: A social-psychological analysis of the nuclear arms race. Journal of Conflict Resolution, 29, 363-389.

Read, S. J., Jones, D. K., \& Miller L. C. (1990). Traits as goal-based categories: The importance of goals in the coherence of dispositional categories. Journal of Personality and Social Psychology, 58, 1048-1061.

Read, S. J., \& Miller, L. C. (1998). On the dynamic construction of meaning. In S. J. Read \& L. C. Miller (Eds.), Connectionist models of social reasoning and social behavior (pp. 27-68). Mahwah, NJ: Lawrence Erlbaum Associates, Inc.

Roberts, B. W., \& Robins, R. W. (2000). Broad dispositions, broad aspirations: The intersection of personality traits and major life goals. Personality and Social Psychology Bulletin, 26, 1284-1296.

Schaller, M., \& Conway, L. G., III (1999). Influence of impression-management goals on the emerging contents of group stereotypes: Support for a social-evolutionary process. Personality and Social Psychology Bulletin, 25, 819-833.

Schaller, M., \& Conway, L. G. (2001). From cognition to culture: The origin of stereotypes that really matter. In G. B. Moskowitz (Ed.), Cognitive social psychology. (pp. 163-176). Mahwah, NJ: Lawrence Erlbaum Associates, Inc.

Schaller, M., Conway, L. G., III, \& Tanchuk, T. L. (2002). Selective pressures on the once and future contents of ethnic stereotypes: Effects of the communicability of traits. Journal of Personality and Social Psychology, 82, 861-919.

Tesser, A., \& Achee, J. (1994). Aggression, love, conformity, and other social psychological catastrophes. In R. R. Vallacher \& A. Nowak (Eds), Dynamical systems in social psychology. (pp. 95-109). San Diego, CA: Academic.

Tooby, J., \& Cosmides, L. (1992). Psychological foundations of culture. In J. Barkow, L. Cosmides, \& J. Tooby (Eds.), The adapted mind (pp. 19-136). New York: Oxford.

VanLange, P., DeBruin, E., Otten, W., \& Joireman, J. (1997). Development of prosocial, individualistic, and competitive orientations: Theory and preliminary evidence. Journal of Personality and Social Psychology, 73, 733-746.

Warner, R. R. (1984). Mating behavior and hermaphroditism in coral reef fishes. American Scientist, 72, 128-136.

Zeeman, E. C. (1976). Catastrophe theory. Scientific American, 234, $65-70 ; 75-83$.

Zeifman, D., \& Hazan, C. (1997). Attachment: The pair in pair-bonds. In J. A. Simpson \& D. T. Kenrick (Eds.), Evolutionary social psychology (pp. 237-264). Mahwah, NJ: Lawrence Erlbaum Associates, Inc. 
Copyright of Personality \& Social Psychology Review is the property of Lawrence Erlbaum Associates and its content may not be copied or emailed to multiple sites or posted to a listserv without the copyright holder's express written permission. However, users may print, download, or email articles for individual use. 